\title{
Design of Reconfigurable Slot Antennas
}

\author{
Dimitrios Peroulis, Member, IEEE, Kamal Sarabandi, Fellow, IEEE, and Linda P. B. Katehi, Fellow, IEEE
}

\begin{abstract}
In this paper the design of a compact, efficient and electronically tunable antenna is presented. A single-fed resonant slot loaded with a series of PIN diode switches constitute the fundamental structure of the antenna. The antenna tuning is realized by changing its effective electrical length, which is controlled by the bias voltages of the solid state shunt switches along the slot antenna. Although the design is based on a resonant configuration, an effective bandwidth of 1.7:1 is obtained through this tuning without requiring a reconfigurable matching network. Four resonant frequencies from 540-890 $\mathrm{MHz}$ are selected in this bandwidth and very good matching is achieved for all resonant frequencies. Theoretical and experimental behavior of the antenna parameters is presented and it is demonstrated that the radiation pattern, efficiency and polarization state of the antenna remain essentially unaffected by the frequency tuning.
\end{abstract}

Index Terms-Electronic tuning, miniaturization, PIN diode switch, reconfigurable antenna, resonant antenna.

\section{INTRODUCTION}

$\mathbf{W}$ ITH ever-increasing demand for reliable wireless communications, the need for efficient use of electromagnetic spectrum is on the rise. In modern wireless systems spread spectrum signals are used to suppress the harmful effects of the interference from other users who share the same channel (bandwidth) in a multiple-access communication system and the selfinterference due to multipath propagation. Also spread spectrum signals are used for securing the message in the presence of unintended listeners and alleviating the effects of communication jammers. One common feature of spread spectrum signals is their relatively high bandwidth. This is specifically true for frequency-hopped spread spectrum communications system. In a frequency-hopped spread spectrum system a relatively large number of contiguous frequency slots spread over a relatively wide bandwidth are used to transmit intervals of the information signal. The selection of the frequency slots for each signal interval is according to a pseudo-random pattern known to the receiver.

Manuscript received October 23, 2000; revised July 18, 2001. This work was supported in part by the U.S. Army Research Office under Grant DAAD19-99-10197 and in part by the MURI MARRS program under Grant 2001-0694-02.

D. Peroulis was with the Radiation Laboratory, Electrical Engineering and Computer Science Department, University of Michigan, Ann Arbor, MI 48109-2122, USA. He is now with the School of Electrical and Computer Engineering, Purdue University, West Lafayette, IN 47906 USA (e-mail: dperouli@purdue.edu).

K. Sarabandi is with the Radiation Laboratory, Electrical Engineering and Computer Science Department, University of Michigan, Ann Arbor, MI 481092122 USA (e-mail: saraband@eecs.umich.edu).

L. P. B. Katehi was with the Radiation Laboratory, Electrical Engineering and Computer Science Department, University of Michigan, Ann Arbor, MI 481092122 USA. She is now with the College of Engineering, Purdue University, West Lafayyete, IN 47907 USA (e-mail: katehi@ purdue.edu).

Digital Object Identifier 10.1109/TAP.2004.841339
Signal propagation over large distances and in urban and forested environment can take place at UHF and lower frequencies. At these frequencies, the size of broadband and efficient antennas is considerable. Techniques used to make the antenna size small, usually renders narrow-band antennas. To make miniature size antennas compatible for a frequency-hopped spread spectrum system, we may consider a reconfigurable narrow-band antenna that follows the pseudo-random pattern of the frequency-hopped modulation. In this paper the design aspects of compact, planar, and reconfigurable antennas are considered and the feasibility of such designs is demonstrated by constructing and testing a planar reconfigurable slot antenna operating at UHF.

Compared to broadband antennas, reconfigurable antennas offer the following advantages: 1) compact size; 2 ) similar radiation pattern and gain for all designed frequency bands; and 3) frequency selectivity useful for reducing the adverse effects of co-site interference and jamming.

In recent years, reconfigurable antennas have received significant attention for their applications in communications, electronic surveillance and countermeasures by adapting their properties to achieve selectivity in frequency, bandwidth, polarization and gain. In particular, preliminary studies have been carried out to demonstrate electronic tunability for different antenna structures [1]-[11]. It has been shown that the operating frequency or bandwidth of resonant antennas can be varied when a tuning mechanism is introduced. Several interesting approaches are presented by Sengupta [1], [2] and Guney [3]. In the literature, tuning is accomplished using varactor diodes [4], [5], or by the application of electrically [6] and magnetically tunable substrates [7], [8] with the use of barium strontium titanate (BST) and ferrite materials, respectively.

Tuning of printed dipole or slot antennas has also been considered since they share the same advantages of portability, low profile and compatibility in integration with other monolithic microwave integrated circuits (MMICs). Kawasaki and Itoh [9] presented a $1 \lambda$ slot tunable antenna loaded with reactive FET components. Although the radiation pattern properties were preserved in all resonant frequencies, the tuning range of the resulting antenna was very limited. Second-resonance cross slot antennas were also presented by Forman et al. [10] in a mixer/phase detector system. A varactor diode was used in the microstrip feed-line and the resonance could be electronically tuned over a $10 \%$ bandwidth. The bandwidth was increased to $45 \%$ when mechanical tuning was used by varying the feed-line length. Dipole tunable antennas were proposed by Roscoe et al. [11] where printed dipoles in series with PIN diodes were studied. The dipole length was varied from $\lambda / 2$ to $1 \lambda$ depending on whether the diodes were OFF or ON. The 
operating frequencies were selected from 5.2 to $5.8 \mathrm{GHz}$, while matching of only $4-5 \mathrm{~dB}$ was achieved.

The slot antenna proposed in this paper utilizes shunt switches that effectively change its electrical length over a very wide bandwidth. To demonstrate the technique a reconfigurable slot antenna capable of operating at four different resonant frequencies over a bandwidth of 1.7:1 is designed and tested. Measurements of the return loss indicate that excellent impedance match can be obtained for all selected resonant frequencies. No especial matching network is used and the matching properties are solely determined by the placement of the switches. The loading effect of the PIN diodes in the antenna is also characterized by a full wave analysis and transmission line theory and comparisons between the real and ideal switches are also studied. Per design goals, it is demonstrated that the reconfigurable slot antenna has the same radiation pattern at all frequencies. Also, the measured radiation patterns agree with the theoretical ones. The polarization characteristics and the efficiency behavior of the antenna as a function of frequency are investigated using both theoretical and experimental data. Finally, some design guidelines are provided and possible design improvements are discussed.

The strict requirements of a constant input impedance, gain, radiation pattern and polarization can only be met, if both the passive structure and the tuning mechanism are carefully designed and effectively integrated into the final design. Therefore, these issues are discussed separately. Section II focuses on the passive antenna structure and its properties. The switching mechanism, its loading effect on the antenna and the final reconfigurable antenna are discussed in Section III. Finally, the measured results are presented in Section IV.

\section{Passive Antenna Design}

The antenna size at UHF and lower becomes critical and therefore special consideration is required. A compact planar geometry is best suited since three-dimensional large and bulky structures are in general undesirable, especially for military applications. Furthermore, some miniaturization techniques have been applied to reduce the size. This section focuses on the passive slot antenna design issues emanating from the above principles.

First, the miniaturization capabilities provided by a high dielectric constant substrate were investigated. Inasmuch as an accurate characterization of its effect is needed, a commercially available moment method code [12] was employed. First, simple slot antennas were simulated at $600 \mathrm{MHz}$ and their resonant lengths were determined as a function of the substrate thickness and dielectric constant (Fig. 1). This analysis suggests that even at low frequencies where the substrate is very thin compared to the wavelength, a miniaturization factor of about 2:1 is possible, if a high dielectric constant substrate is employed. However, the standard commercially available substrates are electrically thin at UHF and below and therefore the 2:1 factor seems to be a limit difficult to exceed even for substrate permittivities as high as 10 .

In an effort to further decrease the total area occupied by the antenna, the slot configuration was altered from its stan-

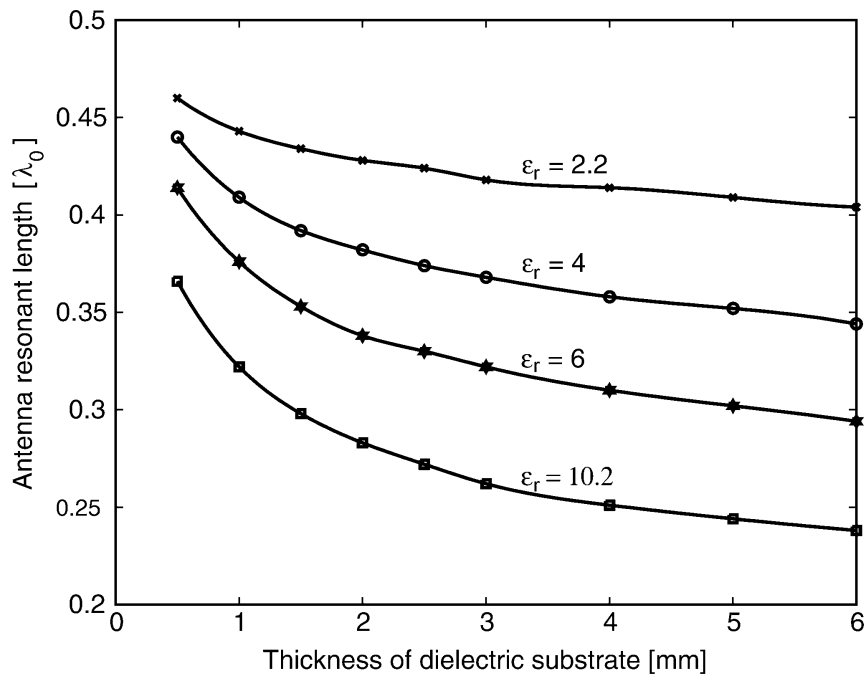

Fig. 1. Resonant length at $600 \mathrm{MHz}$ for straight slot antenna (in free-space wavelength) as a function of substrate thickness and dielectric constant. (a)

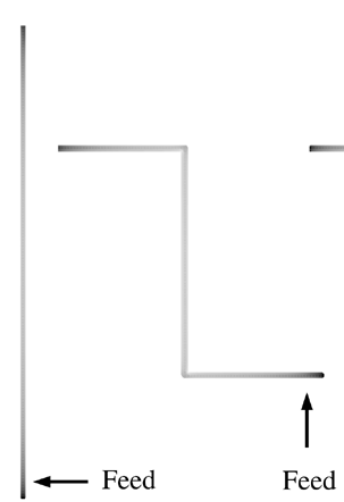

(c)

(d)

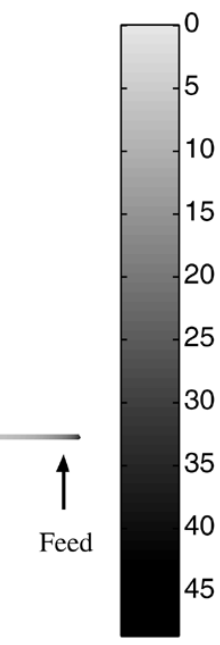

Fig. 2. Computed magnetic current distribution on (a) $600 \mathrm{MHz}$ straight slot antenna, (b) $600 \mathrm{MHz}$ S-shape slot antenna, (c) $700 \mathrm{MHz}$ S-slot, and (d) $600 \mathrm{MHz}$ S-slot with a short-circuit $21 \mathrm{~mm}$ above its bottom edge (scale is in decibels).

dard straight form to an S-shape. From the simulated equivalent magnetic current distribution on the straight and S-shape slots [Fig. 2, antennas (a) and (b)], it is obvious that they both closely follow a sinusoidal pattern with the maximum current concentrated in the middle of the slot. As a result, the two antennas share very similar properties and they only differ in their polarization orientation. Antenna (a) is horizontally polarized, while antenna (b) slant linear polarized. Other more complicated geometrical shapes can also be used, but the S-shape slot does not contain any segments supporting opposing currents, which would considerably deteriorate the radiation efficiency. It should also be mentioned that, although the total area of the antenna is greatly reduced by this geometrical change, the resonant length remains almost unchanged. For example, a resonant length of $136 \mathrm{~mm}$ for a straight slot is slightly increased to $139 \mathrm{~mm}$ for S-slot at $600 \mathrm{MHz}$ for a substrate with $\epsilon_{r}=10.2$ and thickness of $2.54 \mathrm{~mm}$. 


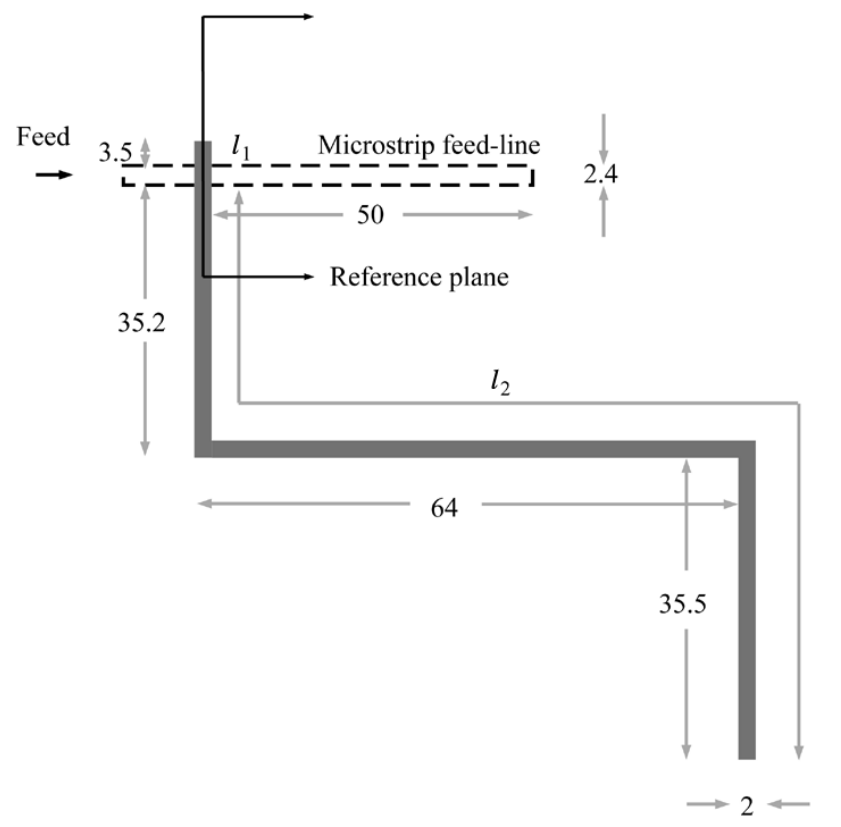

(a)

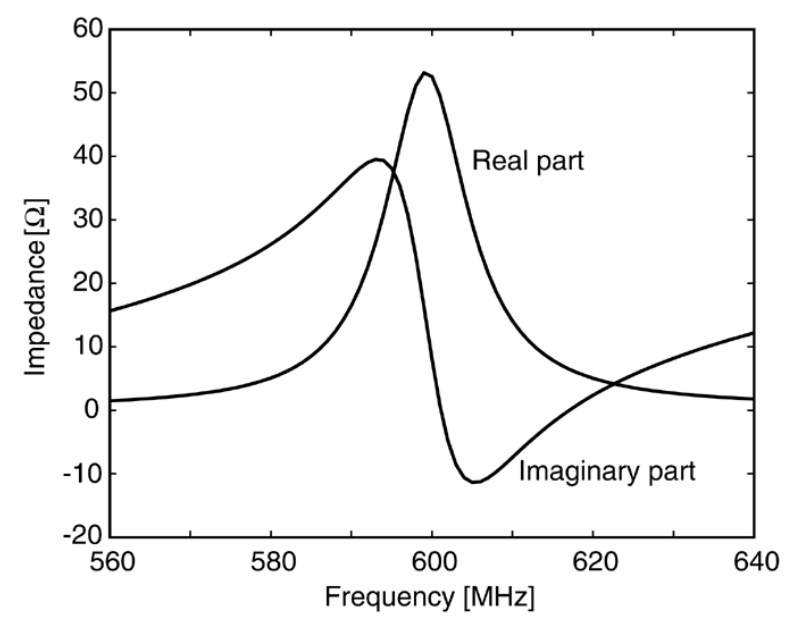

(b)

Fig. 3. (a) S-slot antenna with microstrip feed-line and (b) the real and imaginary parts of the input impedance as a function of frequency. All units are in millimeters. The substrate dielectric permittivity and thickness are 10.2 and $2.54 \mathrm{~mm}$, respectively.

The standard microstrip feed for the simple slot can also be used for the S-shape slot. Fig. 3(a) shows the slot antenna with its feedline, while Fig. 3(b) presents the input impedance at the feeding point as a function of frequency. To achieve a good match to a 50 $\Omega$ line, the microstrip feed-line has to be moved close to one end of the slot antenna. This implies that the antenna input impedance is not very sensitive to small changes in the length of the longer segment $\left[l_{2}\right.$, see Fig. 3(a)]. This property will greatly simplify the design of the tunable slot and its feeding network and will result in minimum complexity and maximum reliability for the final antenna. More details on this issue can be found in Section III. This property of the slot antenna makes it an attractive choice as a reconfigurable structure, since most other antennas(such as dipoles) would require a specially designed matching network.

The resonant frequency of the above structure can be tuned by changing the electrical length of the slot. This may be readily accomplished by introducing a short circuit at a specific location.

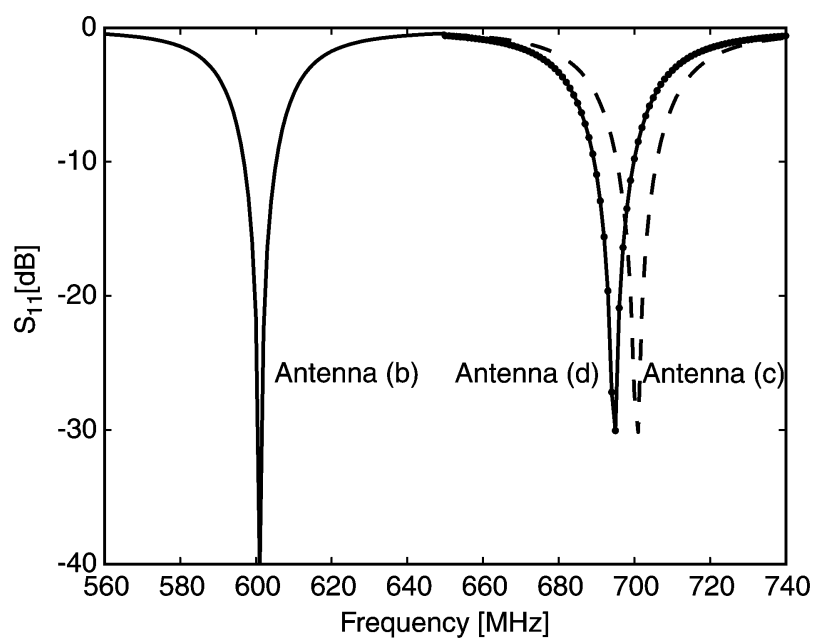

Fig. 4. Simulated results for the return loss of the S-shape slot antennas presented in Fig. 2.

Then the slot will appear to be shorter and therefore the antenna will resonate at a higher frequency. The three $\mathrm{S}$-shape slots in Fig. 2 demonstrate these concepts. Slot antenna (b) resonates at $600 \mathrm{MHz}$ with a resonant length of $139 \mathrm{~mm}$. Antenna (c) is $21 \mathrm{~mm}$ shorter and is designed to resonate at $700 \mathrm{MHz}$. Finally, antenna (d) is obtained by modifying (b). In particular, antenna (b) is short circuited at $21 \mathrm{~mm}$ above its lower end. The simulated return losses for these three slots are shown in Fig. 4. It is also important to note that the microstrip feed-line remains unchanged in all three cases. That is, the distance between the top end of the slot and the feed line cross point remains constant and is equal to $3.2 \mathrm{~mm}$. This means that, although the resonant frequency is shifted by $100 \mathrm{MHz}$, very good matching is achieved for both (c) and (d) slot antennas without needing to modify the feeding network. In addition, slot antennas (c) and (d) have almost identical resonant frequencies. The small difference in the resonant frequency comes from the fact that antenna (d) appears somewhat electrically longer than (c) due to the parasitic effects of the short circuit. Therefore, tunability is possible by introducing these short circuits with no special matching network. Although Fig. 2 illustrates the basic concept of reconfigurability on a dual band antenna, it is obvious that it can be extended to antennas with several bands of operation. The number of these bands depends on the number of switches on the antenna. For example, a four band antenna is presented in Section III-C and it is demonstrated that the resonant frequency can be digitally controlled by an array of four switches.

\section{Modeling And Design of Active Antennas}

In the previous section we presented the basic principle of controlling the antenna resonant frequency. It was also shown that even when a perfect short circuit is used, the parasitic effects of the short can slightly affect the antenna performance and particularly the resonant frequency. The parasitic effects become worse when a switch with finite isolation is used. This section addresses the issues related to the design of a suitable solid state switch and on the characterization of its effects on the antenna 


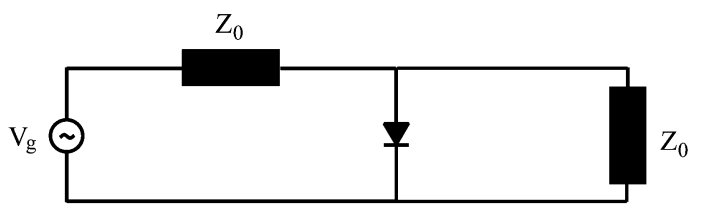

(a)

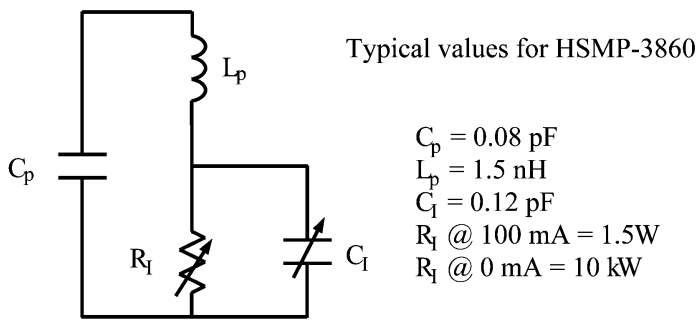

(b)

Fig. 5. (a) PIN diode connected as a shunt switch in a transmission line. (b) RF equivalent circuit for PIN diode including packaging effects.

performance. Finally, the complete reconfigurable antenna design is presented at the end of the section together with its theoretical performance.

\section{A. Switch Design}

To implement the electronic reconfigurability, the ideal shunt switches must be replaced by PIN diodes. PIN diode's reliability, compact size, high switching speed, small resistance and capacitance in the ON and OFF state, respectively, make it most appropriate for the application at hand. The RF equivalent circuit of the diode is shown in Fig. 5(b) for both the ON and OFF states. The reactive components $C_{p}$ and $L_{p}$ model the packaging effect, while the others come from the electric properties of the diode junction in the ON and OFF positions [13]. Typical values are also given for the HSMP-3860 diode [14] used in this paper. The computed isolation (defined as $1 /\left|S_{21}\right|^{2}$ ) for the circuit shown in Fig. 5(a) is given by [14]

$$
\alpha=10 \log \left[\frac{\left(\frac{R_{d} Z_{0}}{R_{d}^{2}+X_{d}^{2}}+2\right)^{2}+\left(\frac{X_{d} Z_{0}}{R_{d}^{2}+X_{d}^{2}}\right)^{2}}{4}\right]
$$

where $Z_{d}=R_{d}+j X_{d}$ is the equivalent impedance of the diode and $Z_{0}$ is the characteristic impedance of the line. In the example considered here, the characteristic impedance of the line is approximately equal to $60 \Omega$, which is calculated by the moment method code [12] for a slotline with a width of $2 \mathrm{~mm}$, a finite ground plane of $60 \mathrm{~mm}$ (on both sides of the slot) and a substrate permittivity $\epsilon_{r}=10.2$ (RT/Duroid) [15].

The switch bias network is presented in Fig. 6. An inductor of $470 \mathrm{nH}$ and three $10 \mathrm{pF}$ capacitors are used to improve the RF-dc signal isolation. These values were chosen based on the bias network RF equivalent circuit shown in Fig. 6(b). The simulated performance for the ON and OFF states is presented in Fig. 6(c). The RF-dc isolation is better than $30 \mathrm{~dB}$ for both states and the return loss is less than $-20 \mathrm{~dB}$ for the OFF state. Finally, the RF-RF isolation is greater than $10 \mathrm{~dB}$ up to $1 \mathrm{GHz}$. High-frequency diodes or RF MEMS switches can provide higher isolation for applications beyond UHF.

\section{B. Switch Loading on the Antenna}

Although the switch isolation is important since it determines the frequency selectivity of the antenna, the switch loading on the antenna is equally important inasmuch as it affects its resonant frequency and input impedance. The loading effects must be taken into account for an accurate prediction of the antenna resonant frequency and input impedance, especially when more than one switch is used for multifrequency operation.

A transmission line equivalent circuit that models the loading effect of one diode on the antenna is shown in Fig. 7. The transverse resonant technique [16] states that

$$
Z_{R}\left(z^{\prime}\right)+Z_{L}\left(z^{\prime}\right)=0
$$

where $Z_{R}\left(z^{\prime}\right)$ and $Z_{L}\left(z^{\prime}\right)$ are the input impedances on the right and left of the reference point, respectively. For the unloaded transmission line in Fig. 7(a) (2) simplifies to

$$
\tan \left(\beta l_{L}\right)+\tan \left(\beta l_{R}\right)=0
$$

or

$$
\beta\left(l_{L}+l_{R}\right)=n \frac{\pi}{2}, \quad n=1,2,3 \ldots
$$

which is the well known formula for these resonant antennas. Now it is important to see what happens in the simplest case of having one switch on the antenna. Fig. 7(b) shows the equivalent circuit of a transmission line loaded with one switch in the OFF position. Equation (2) then becomes

$$
\begin{aligned}
{\left[Z_{0} \omega_{R} C-\cot \left(\beta l_{R 2}\right)\right]\left[\tan \left(\beta l_{L}\right)+\tan \left(\beta l_{R 1}\right)\right] } \\
=1+\tan \left(\beta l_{L}\right) \tan \left(\beta l_{R 1}\right) .
\end{aligned}
$$

Equation (5) can of course be solved numerically and an iterative method can be employed for finding the unknown lengths until the desired resonant frequency $\left(f_{R}\right)$ has been achieved. A similar procedure can be followed if more than one switch is used on the slot, but the process becomes a little more complicated if all resonant frequencies are to be specified. We also need to note that (5) does not include any packaging effects, but these can be readily incorporated in the model, resulting in a more accurate computation.

Although the OFF state loading effects have been only discussed up to now, the small on state resistance also affects the antenna performance and particularly its input impedance. Full wave analysis was used to model these effects. For a first order approximation, the diode resistance was modeled as a thin film resistor on top of the slot and the packaging parasitic elements were neglected in this analysis. The parasitic element effects in the ON state can be important especially at the highest frequencies [see Fig. 5(c)]. Fig. 8(a) shows the simulated geometry of an $\mathrm{S}$-shape slot antenna loaded with a resistive film, which is fed by a microstrip line and Fig. 8(b) shows the simulated return loss versus the switch ON state resistance for four different cases between 0 to $5.6 \Omega$. In all four cases the position of the $50 \Omega$ feed-line was kept unchanged. It is obvious that the matching level deteriorates rapidly as the resistance value increases, and for resistance values above $1.5 \Omega$ the matching level becomes unacceptable. 


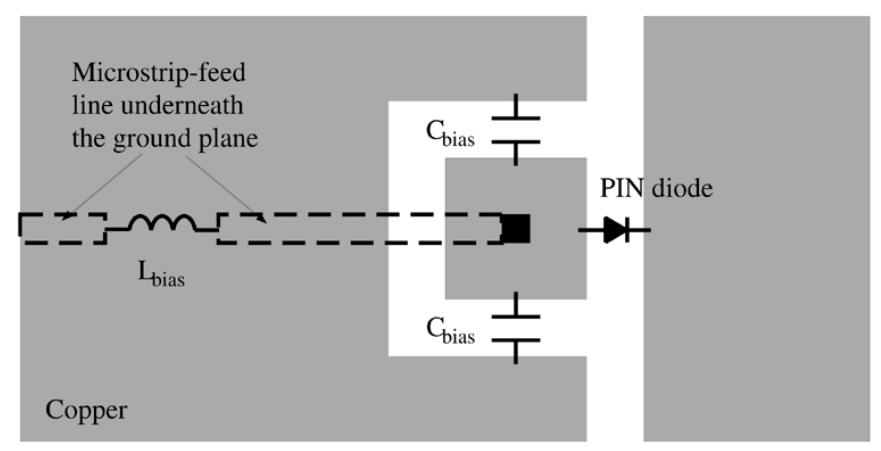

(a)

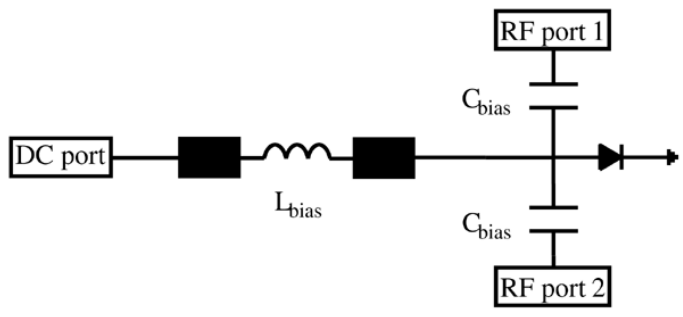

(b)
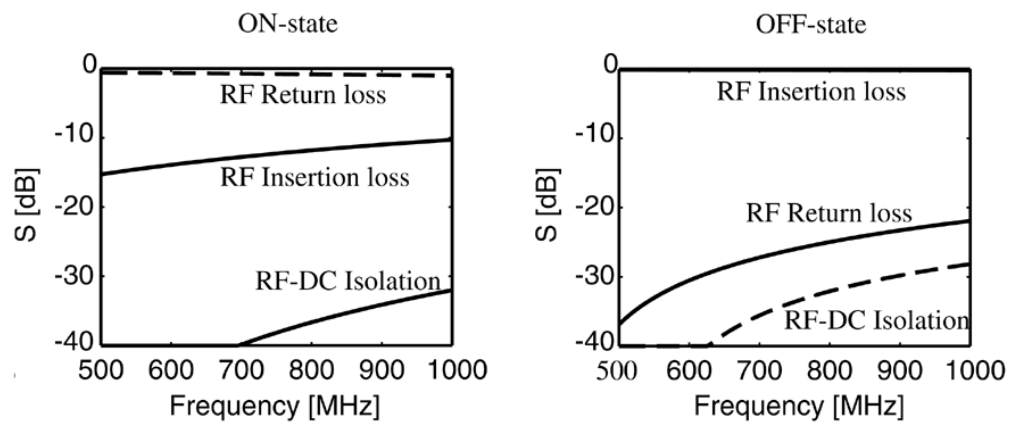

(c)

Fig. 6. (a) Layout of switch biasing network. (b) RF equivalent circuit. (c) On and Off-state simulated RF performance.

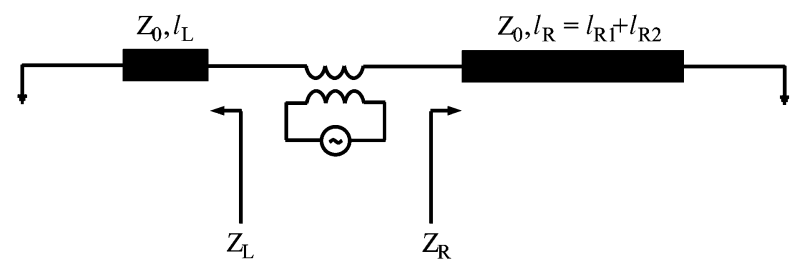

(a)

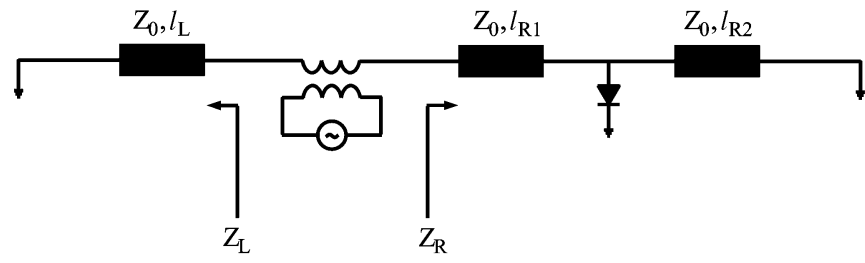

(b)

Fig. 7. RF equivalent circuits for determining the resonant frequency of (a) unloaded and (b) loaded with a single switch slot antenna.

However, this degradation can be avoided to some extent by elongating the upper end of the slot as the resistance is increased. Fig. 8(c) shows the improvement on the antenna matching when the slot length is adjusted. It is found that, in all three cases, only a very small line segment length needs to be added in order to improve the input impedance of the antenna. Even for a resistance value of $5.6 \Omega$ the required line segment length is less than $3 \%$ of the total slot length, resulting in only a small change in the resonant frequency. This method of maintaining a good impedance match will be utilized later for the design of the reconfigurable antenna by placing additional switches (matching-switches) on the slot above the feed-line and synchronizing them together with the switches at the other end of the slot (frequency-switches). However, it should be noted that the matching switches will not represent perfect shorts and they will introduce an extra loading effect. Nonetheless, this effect is negligible and matching levels of better than $-20 \mathrm{~dB}$ can be achieved, as will be seen next. Therefore, the matching properties of the reconfigurable antenna will solely depend on the position of an array of switches on the slot and no matching network will be necessary as frequency changes.

Having discussed the loading effects of the switches on the matching properties of the antenna, their effects on the radiation characteristics of the antenna need to be found as well. Ideally, the radiation efficiency should be that of the half-wavelength dipole, since the antenna behaves effectively as a $\lambda / 2$ resonant slot at each of its operating frequencies. However, the ON state resistance of the switches will obviously result in power dissipation and finally degradation in the antenna efficiency. The dissipated power obviously depends on the diode's ON resistance 


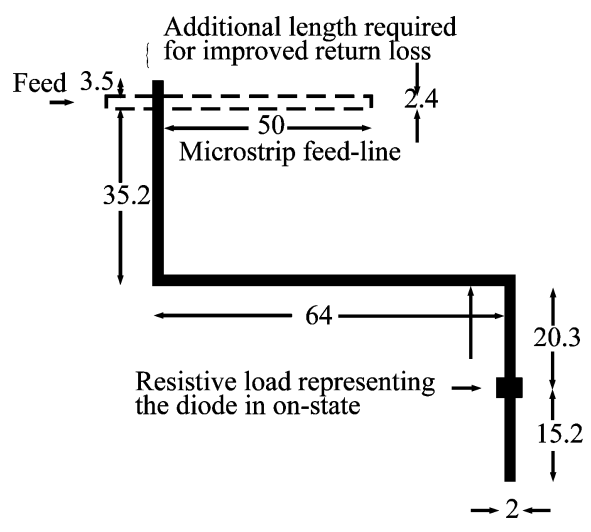

(a)

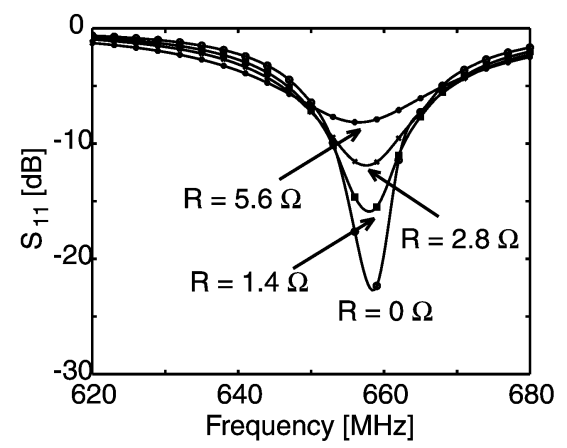

(b)

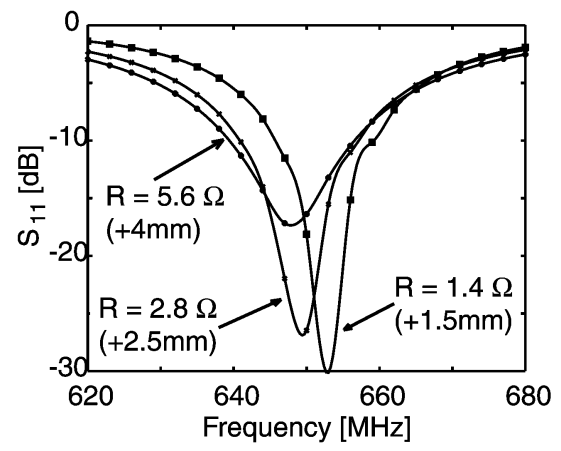

(c)

Fig. 8. (a) Slot antenna with resistive load representing actuated switch (units are in millimeters). (b) Return loss for different values of switch resistance. (c) Improved return loss with minor adjustments $(<4 \mathrm{~mm})$ in the slot length above the feeding point.

and on the number of the switches on the antenna. Table I shows the computed efficiency for the antennas previously discussed in Fig. 8. Dielectric and metallic loss has been included in all cases. This explains the nonideal efficiency when $R=0 \Omega$.

The above antenna efficiency analysis shows that even for a small series resistance of $R=1.4 \Omega$ the antenna gain will be approximately $2.5 \mathrm{~dB}$ lower than that of an ideal half-wavelength dipole. This is an inherent drawback of using PIN diode switches. However, micro-electro-mechanical (MEMS) switches are becoming increasingly important and are now a viable alternative as they offer very low power consumption and they come even in smaller packages. [17], [18]. It has also be shown [20] that capacitive type MEMS switches exhibit very low ohmic losses and therefore can be used for maximized antenna efficiency. However, the required ON capacitance values renders them impractical for UHF frequencies. Hence
TABLE I

COMPUTED EFFICIENCY FOR SLOT ANTENNAS WiTH A Single SWITCH VERSUS ON STATE RESISTANCE VALUE

\begin{tabular}{c|c|c|c|c}
\hline $\mathrm{R}[\Omega]$ & 0 & 1.4 & 2.8 & 5.6 \\
\hline Efficiency [\%] & 71.8 & 55.6 & 45.6 & 33.9 \\
\hline
\end{tabular}

metal-to-metal contact switches [22], which have no cutoff frequency should be considered in such a design.

\section{Final Reconfigurable Antenna Design and Properties}

Based on the previously discussed design principles, a reconfigurable slot antenna design [shown in Fig. 9(a)] is presented here. Four switches are used in order to tune the antenna over a range of 540-950 MHz. Both full wave analysis and the transmission line model were used in the design process. In this design three frequency-switches and a single matching-switch are used. Table II summarizes the calculated resonant frequencies and the conditions of all four switches for each resonant frequency. The transmission line model has the advantage of allowing fast and accurate (as will be proven later) computation of the resonant frequencies and can easily incorporate the diode parasitics. However, the full wave analysis is essential when an accurate prediction of the antenna input impedance is needed. In the moment method code, the diodes were simulated as metal-insulator-metal (MIM) capacitors and as thin film resistors in the OFF and ON states, respectively, and as a result the packaging parasitics were ignored. This explains the $5 \%$ differences observed in the computed resonances between the two models. Fig. 9(b) shows the calculated return loss where a matching level of better than $-20 \mathrm{~dB}$ has been achieved for all the operating frequencies.

Since at every operating frequency the antenna radiates as a $\lambda / 2$ slot, the radiation pattern remains unchanged when the frequency is shifted. The same holds for the antenna directivity. The E and H-planes of a typical calculated pattern are shown in Fig. 9(c). Since the antenna has been designed on a electrically thin substrate (at UHF) the radiation pattern is symmetric on the two sides of the slot. However, the efficiency and the gain will be reduced compared to a half-wavelength dipole due to the resistive losses caused by the diodes. Fig. 9(d) shows the calculated gain using the moment method analysis [12]. The gain is approximately $-1 \mathrm{~dB}$ for the lowest frequencies and increases to about $0.7 \mathrm{~dB}$ for the highest one. Similar results hold for the antenna efficiency.

The reference angle of $0^{\circ}$ in the previous graphs represents the direction normal to the antenna ground plane. Although the $\mathrm{S}$-shape pattern considerably reduces the antenna occupied area, it has the inherent drawback that the polarization does not remain constant as the frequency is changed. However, as Table III shows, the polarization does not change considerably (variation of about $30^{\circ}$ ). This is due to the fact that the antenna polarization (always slant linear) is dominated by the orientation of the middle segment of the slot where most radiated field is emanated from. Therefore, if the orientation of the receiving antenna does not follow that of the transmitter as the frequency is changed, a maximum polarization mismatch of $25 \%$ will be incurred. The orientation of linear polarization reported in Table III is with respect to the $x$ axis [see Fig. 9(a)]. 


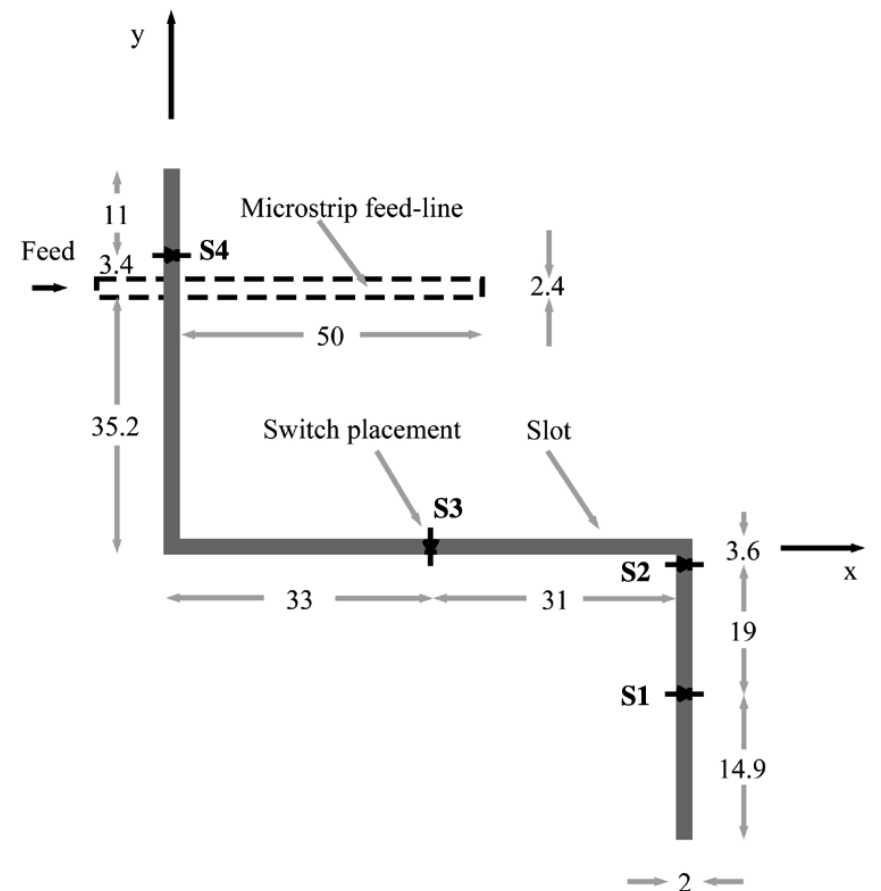

(a)

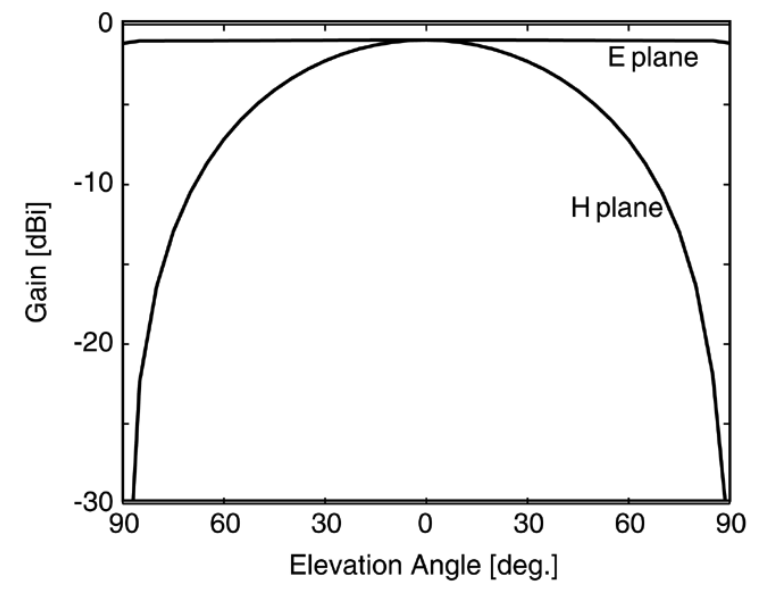

(c)

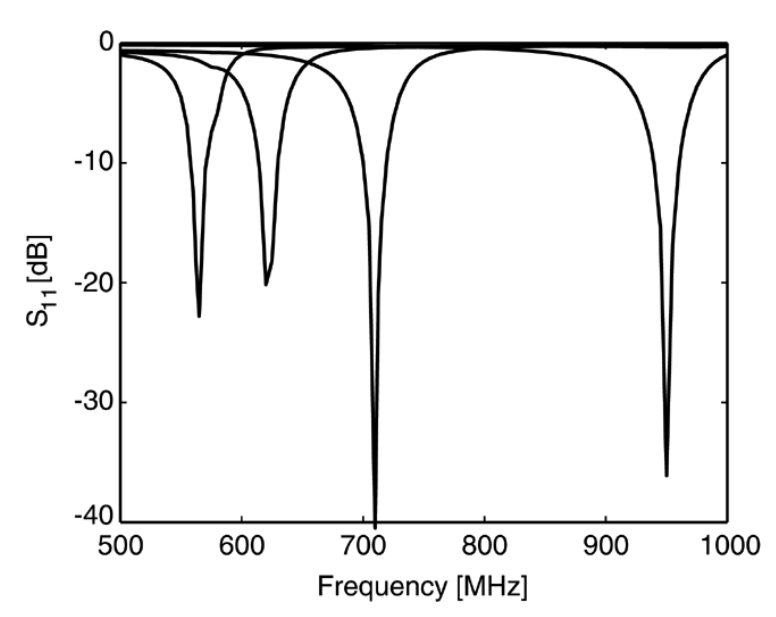

(b)

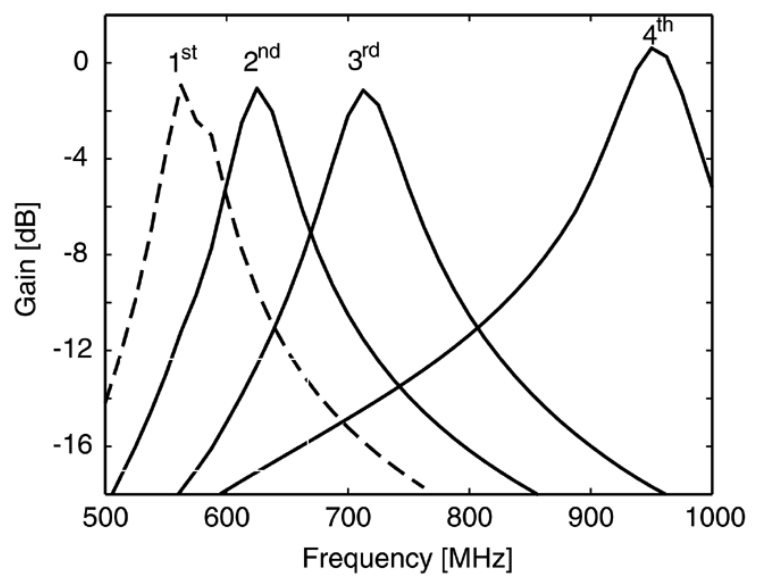

(d)

Fig. 9. (a) Reconfigurable slot antenna (units are in millimeters) (b) Simulated return loss for the four resonant frequencies. (c) Typical radiation pattern. (d) Simulated gain the four resonant frequencies.

TABLE II

Theoretically Calculated Resonant FreQuencies Using Full WaVE ANALYSIS AND TRANSMISSION LINE MODEL

\begin{tabular}{c|c|l}
\hline $\begin{array}{c}f_{R}[\mathrm{MHz}] \\
(\mathrm{TLN})^{a}\end{array}$ & $\begin{array}{c}f_{R}[\mathrm{MHz}] \\
(\mathrm{MM})^{b}\end{array}$ & Switch Configuration \\
\hline 542 & 561 & $4=$ ON 1,2,3 = OFF \\
\hline 596 & 627 & $1,4=$ ON $2,3=$ OFF \\
\hline 688 & 711 & $2,4=$ ON $1,3=$ OFF \\
\hline 1002 & 950 & $3=$ ON $1,2,4=$ OFF \\
\hline
\end{tabular}

\section{MEASUREMENTS AND DISCUSSION}

The reconfigurable antenna designed in the previous section was fabricated on a 100 mil thick RT/Duroid substrate $\left(\epsilon_{r}=\right.$ 10.2). The size of the ground plane was $5 \times 5 \mathrm{in}^{2}$.

The first task was to measure the resonances and an HP8753D vector network analyzer was used for the S-parameter measure-
TABLE III

Calculated Polarization for the Reconfigurable Antenna

\begin{tabular}{c|c|c|c|c}
\hline$f_{R}[\mathrm{MHz}]$ & 561 & 627 & 711 & 950 \\
\hline Angle $\left(^{\circ}\right)$ & 60 & 70 & 60 & 40 \\
\hline
\end{tabular}

ments. The biasing voltage for the switches was provided by a dc voltage source. After calibrating the network analyzer the antenna return loss was measured when different combinations of the switches were activated. The measured data are presented in Fig. 10, where a return loss of better than $-13 \mathrm{~dB}$ is observed at all resonances. The measured resonances are shown in Table IV together with the necessary biasing conditions. Satisfactory agreement between theoretical, Table II, and experimental, Table IV, data is observed. In addition, the transmission line model gives slightly better results-except the highest frequency-mainly because the parasitic reactive elements have been included in this model and not in the moment method tech- 


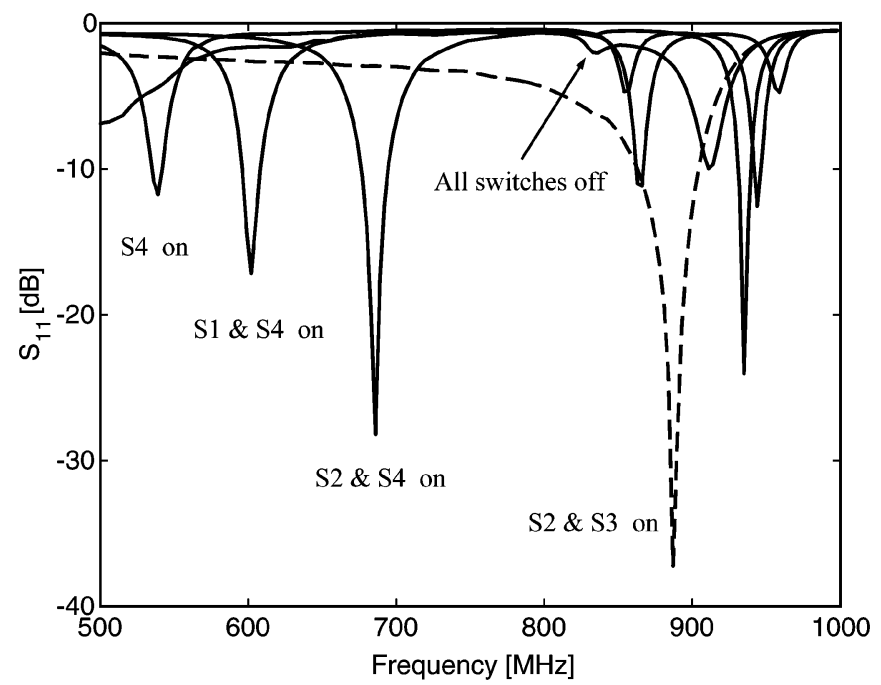

Fig. 10. Measured resonant frequencies of the reconfigurable antenna.

TABLE IV

MEASURED RESONANT FREQUENCIES AND THE NECESSARY BIAS VOLTAGES FOR THE SWITCHES

\begin{tabular}{c|c|c|c|c}
\hline \multirow{2}{*}{$f_{R}[\mathrm{MHz}]$} & \multicolumn{4}{|c}{ Bias Voltage [V] } \\
\cline { 2 - 5 } & S1 & S2 & S3 & S4 \\
\hline \hline 537 & -20 & -20 & -20 & 1.1 \\
\hline 603 & 1.1 & -20 & -20 & 1.1 \\
\hline 684 & 0 & 1.1 & -20 & 1.1 \\
\hline 887 & 0 & 1.1 & 1.1 & 0.2 \\
\hline
\end{tabular}

nique. However, this is not true for the highest resonant frequency where an error of $13 \%$ exist between the transmission line model and the measurement. This discrepancy can be attributed to the fact that the properties of the diodes, and particularly the element values of its equivalent circuit, cannot be assumed constant up to $1 \mathrm{GHz}$.

A reverse voltage of $-20 \mathrm{~V}$ was applied to maintain the switches in the OFF position and by doing so a better matching level was achieved. This is an important issue particularly when the antenna is used as the transmitter. Since the structure is a resonant structure strong electric fields are established that can turn the diodes ON and OFF at the RF frequency and ruin the small signal design. This effect was clearly observed at the lowest resonance with an input power of $0 \mathrm{dBm}$. In this case an improvement of about $5 \mathrm{~dB}$ was achieved by changing $0 \mathrm{~V}$ bias to $-20 \mathrm{~V}$.

One more interesting effect was observed for the highest resonance. We noticed that better matching level would occur, if not only S3 but also S2 was forward biased. This is due to the relatively low isolation that each diode provides at these relatively high frequencies [see Fig. 5(c)]. Therefore, biasing S2 results in higher isolation and reduces the effect of leaked magnetic current in the area after the switch. The improvement in the return loss was approximately $10 \mathrm{~dB}$ compared to leaving S2 unbiased for this frequency.

Next, far field patterns were measured in the University of Michigan's anechoic chamber. The $\mathrm{E}$ and $\mathrm{H}$ plane were measured as well as the corresponding cross-polarization for each operating frequency. An RF signal and a dc voltage source were used with the reconfigurable antenna and a dipole with adjustable length was employed as the receiving antenna. The dipole length was appropriately adjusted for each operating frequency of the transmitting antenna until maximum received power was recorded. In order to find the E plane, the transmitting antenna was rotated until the electric field was vertically polarized. Then the transmitter, placed on a turn table, was azimuthally rotated for measuring $\mathrm{E}$ plane cuts. The cross-polarized pattern was measured by rotating the receiver antenna by $90^{\circ}$. H plane pattern measurements were conducted in a similar manner.

Although for slot antennas printed on a substrate it is expected that the radiated power be higher in the half-space that include the dielectric substrate, no appreciable difference was observed experimentally. This is easily explained since in this case the dielectric thickness is about $\lambda / 200$ at $600 \mathrm{MHz}$ and the size of the ground plane is small (approximately $\lambda / 3$ ) at the same frequency. Therefore, the antenna is almost bi-directional and equivalent to a dipole in free space.

The measured data are presented in Fig. 11 for each resonant frequency. In these plots $0^{\circ}$ denote the direction of maximum radiated power. These measurements show that the $\mathrm{H}$ plane closely follows the expected sinusoidal pattern. However, some slight asymmetries near $\pm 90^{\circ}$ exist for almost all frequencies. These discrepancies originate primarily from two sources. First, parasitic radiation from the cables and the feeding network and second, radiation from the edges of the dielectric. These sources of radiation also affected the E plane pattern measurements and they caused a difference of 3-4 $\mathrm{dB}$ between the minimum and maximum measured value (see Fig. 11). Despite these discrepancies, it is clear that the far-field pattern remains unchanged versus the frequency tuning.

Gain measurements are accomplished using the comparison method [24]. A log-periodic antenna with $6 \mathrm{dBi}$ gain at 600 $\mathrm{MHz}$ was used as a reference antenna for these measurements. The second resonance at $593 \mathrm{MHz}$ was chosen as the operating frequency of the reconfigurable antenna, so that to make direct comparisons with the reference antenna possible. To measure the gain, the power received by the receiver dipole at 593 $\mathrm{MHz}$ was recorded when both the reference and the reconfigurable antennas were used in the transmitting mode inside the anechoic chamber under the same conditions. The measured gain was found $-1.1 \mathrm{dBi}$, which corresponds to an efficiency of $47 \%$. These results closely resemble the calculated data. It should also be pointed out that the gain of the slot antenna is reduced not only from the forward-biased diode resistance, but also from the small ground plane size. However, a comparison between the measured and calculated data reveals that the dominant degrading factor in gain is the dissipated power on the diodes rather the ground plane size.

Finally, the antenna polarization was measured and the method previously described for the pattern measurement was employed. The measured polarization orientation at each frequency is provided in Table V. As discussed before, although the polarization does not remain absolutely constant as the frequency is changed, the variation range is small and comparable to the theoretical data (see Table III). 


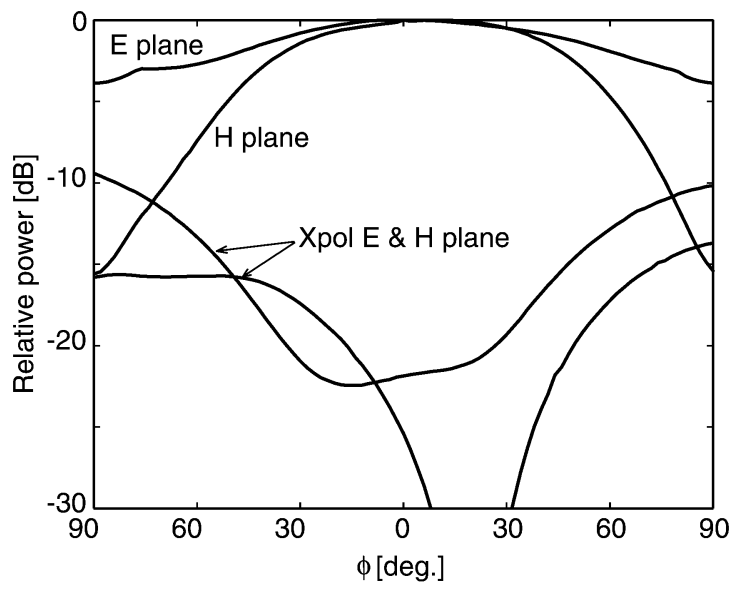

(a) $\mathrm{f}=537 \mathrm{MHz}$

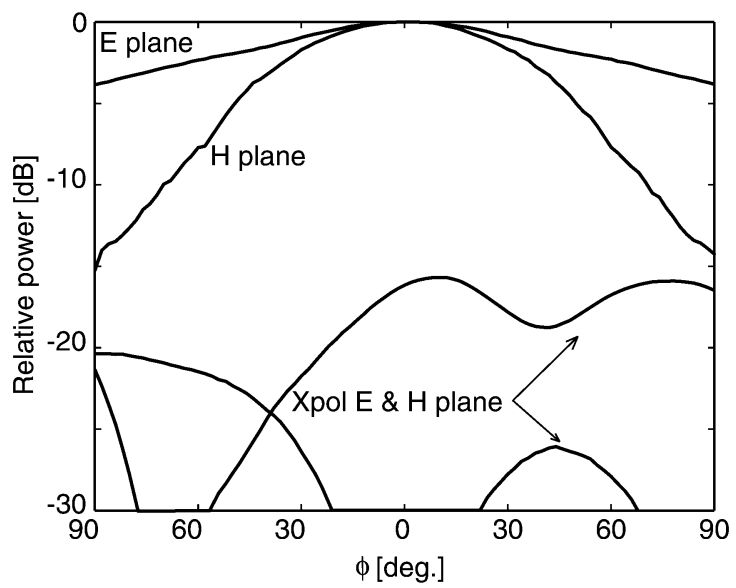

(c) $\mathrm{f}=684 \mathrm{MHz}$

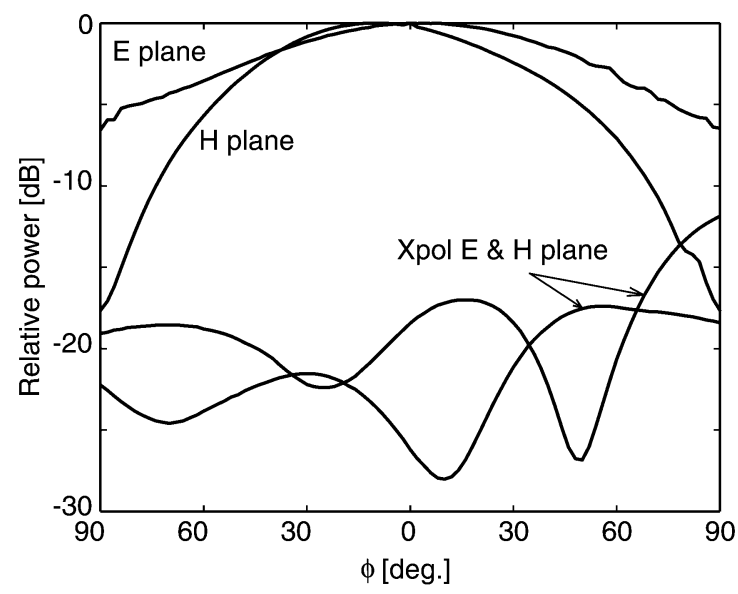

(b) $\mathrm{f}=603 \mathrm{MHz}$

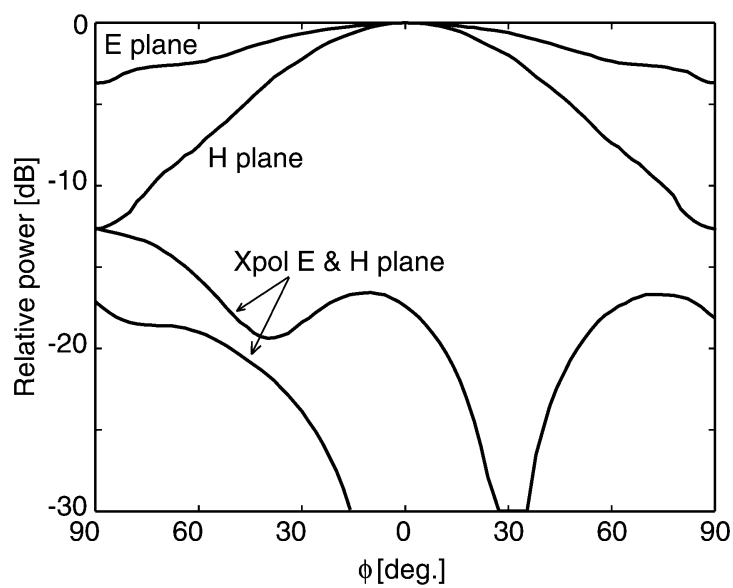

(d) $\mathrm{f}=887 \mathrm{MHz}$

Fig. 11. Measured radiation patterns for the four resonant frequencies.

TABLE V

MEASURED PolariZATION FOR THE RECONFIGURABLE ANTENNA

\begin{tabular}{c|c|c|c|c}
\hline$f_{R}[\mathrm{MHz}]$ & 537 & 603 & 684 & 887 \\
\hline Angle $\left(^{\circ}\right)$ & 57 & 70 & 55 & 33 \\
\hline
\end{tabular}

\section{CONCLUSION}

A novel method for designing affordable, compact, reconfigurable antennas is proposed in this paper. This method relies on changing the effective length of a resonant slot antenna by controlling combinations of electronic RF switches. Theoretical results for significant antenna parameters were validated experimentally. Important issues involved in the design of such antennas and guidelines were also discussed. Based on the proposed method, a compact planar reconfigurable slot antenna was designed, fabricated and measured and a tuning range of 1.7:1 in the operating frequency was demonstrated. Although such a broad range was achieved, no matching network was required for the antenna. Another salient feature of this design, backed by theory and experiments, is that the radiation characteristics of this antenna remain essentially unaffected by the frequency tuning. The design procedure is general enough and allows even wider tuning ranges to be achieved. By employing suitable switches it can be also readily extended to higher frequency commercial and military applications.

\section{REFERENCES}

[1] D. L. Sengupta, "Resonant frequency of a tunable rectangular patch antenna," Electron. Lett., vol. 20, no. 15, pp. 614-615, Jul. 1984.

[2] - "The transmission line model for rectangular patch antennas," in IEEE Antennas and Propagation Symp. Dig., vol. 21, 1983, pp. 158-161.

[3] K. Guney, "Resonant frequency of a tunable rectangular microstrip patch antenna," Microw. Opt. Technol. Lett., vol. 7, no. 12, pp. 581-585, Aug. 1994.

[4] N. Fayyaz, S. Safavi-Naeini, E. Shin, and N. Hodjat, "A novel electronically tunable rectangular patch antenna with one octave bandwidth," in Proc. IEEE Canadian Conf. Electrical and Computer Engineering, vol. 1, 1998, pp. 25-8.

[5] S. H. Al-Charchafchi and M. Frances, "Electronically tunable microstrip patch antennas," in IEEE Antennas and Propagation Symp. Dig., vol. 1, 1998, pp. 304-7.

[6] K. A. Jose, V. K. Varadan, and V. V. Varadan, "Experimental investigations on electronically tunable microstrip antennas," Microw. Opt. Technol. Lett., vol. 20, no. 3, pp. 166-169, Feb. 1999.

[7] P. J. Rainville and F. J. Harackewiez, "Magnetic tuning of a microstrip patch antenna fabricated on a ferrite film," IEEE Microw. Guided Wave Lett., vol. 2, no. 12, pp. 483-485, Dec. 1992.

[8] R. K. Mishra, S. S. Pattnaik, and N. Das, "Tuning of microstrip antenna on ferrite substrate," IEEE Trans. Antennas Propag., vol. 41, no. 2, pp. 230-233, Feb. 1993.

[9] S. Kawasaki and T. Itoh, "A slot antenna with electronically tunable length," in IEEE Antennas and Propagation Symp. Dig., vol. 1, 1991, pp. $130-3$.

[10] M. A. Forman and Z. B. Popovic, "A tunable second-resonance crossslot antenna," in IEEE Antennas and Propagation Symp. Dig., vol. 1, 1997, pp. 18-21.

[11] D. J. Roscoe, L. Shafai, A. Ittipiboon, M. Cuhaci, and R. Douville, "Tunable dipole antennas," in IEEE Antennas and Propagation Symp. Dig. vol. 2, 1972, pp. 672-5.

[12] “Zeland's IE3D, Release 7,”, 2000. 
[13] "Linear Models for Active Diode Surface Mount Packages," Hewlett Packard, Application Note 1124.

[14] “Applications of PIN Diodes,” Hewlett Packard, Application Note 922.

[15] Rogers Microwave Products.

[16] C. Balanis, Advanced Engineering Electromagnetics. New York: Wiley, 1989, ch. 8

[17] C. Goldsmith, J. Randall, S. Eshelman, T. H. Lin, D. Denniston, S. Chen, and B. Norvell, "Characteristics of micromachined switches at microwave frequencies," in IEEE Microwave Theory and Techniques Symp. Dig., vol. 2, 1996, pp. 1141-4.

[18] S. Pacheco, L. P. B. Katehi, and C. T. Nguyen, "Design of low actuation RF MEMS Switch," in IEEE Microwave Theory and Techniques Symp. Dig., vol. 1, 2000, pp. 165-170.

[19] J. B. Muldavin and G. M. Rebeiz, "High-isolation CPW MEMS shun switches-part 1: Modeling," IEEE Trans. Microw. Theory Tech., vol. 48, no. 6, pp. 1045-1052, Jun. 2000.

[20] — "High-isolation CPW MEMS shunt switches-part 2: Design," IEEE Trans. Microw. Theory Tech., vol. 48, no. 6, pp. 1053-1056, Jun. 2000

[21] D. Peroulis, S. P. Pacheco, and L. P. B. Katehi, "RF MEMS switches with enhanced power-handling capabilities," IEEE Trans. Microw. Theory Tech., vol. 51, no. 1, pp. 59-68, Jan. 2004.

[22] J. J. Yao and M. F. Chang, "A surface micromachined miniature switch for telecommunications applications with signal frequencies from DC up to $4 \mathrm{GHz}$, in Proc. Int. Solid-State Sensors and Actuators Conf.-TRANSDUCERS, vol. 2, 1995, pp. 25-9.

[23] D. Hyman and M. Mehregany, "Contact physics of gold microctantcs for MEMS switches," IEEE Trans. Compon. Packag. Technol., vol. 22, no. 3, pp. 357-364, Sep. 1999.

[24] W. L. Stutzman and G. A. Thiele, Antenna Theory and Design, 2nd ed. New York: Wiley, 1998, ch. 9.

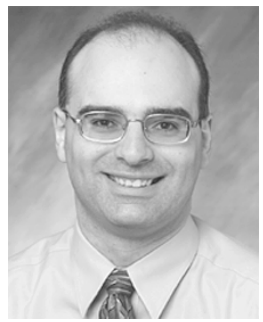

Dimitrios Peroulis (S'99-M'04) was born in Athens, Greece, in 1975. He received the Diploma degree in electrical and computer engineering from the National Technical University of Athens, in 1993 and the M.S.E. and Ph.D. degrees in electrical engineering from The University of Michigan at Ann Arbor, in 1999 and 2003, respectively.

Since August 2003, he has been an Assistant Professor at the School of Electrical and Computer Engineering at Purdue University, West Lafayette, IN His current research work is focused on microelectromechanical systems (MEMS) for multifunctional wireless communications systems.

Dr. Peroulis was the recipient of the 2002 Rackham Graduate School pre-doctoral fellowship presented by The University of Michigan at Ann Arbor and the Third Place Award at the Student Paper Competition presented at the 2001 IEEE MTT-S International Microwave Symposium, Phoenix, AZ. He also received two Student Paper Awards (honorable mentions) at the Student Paper Competitions presented at the 2001 IEEE AP-S International Symposium, Boston, MA and the 2002 IEEE MTT-S International Microwave Symposium, Seattle, WA

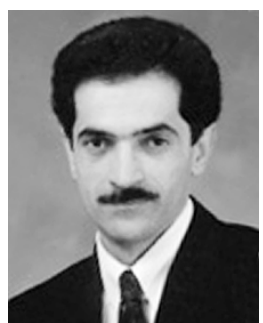

Kamal Sarabandi (S'87-M'90-SM'92-F'00) received the B.S. degree in electrical engineering from Sharif University of Technology, Tehran, Iran, in 1980, the M.S. degree in electrical engineering/mathematics, and the Ph.D. degree in electrical engineering from The University of Michigan-Ann Arbor, in 1986 and 1989, respectively.

$\mathrm{He}$ is Director of the Radiation Laboratory and a Professor in the Department of Electrical Engineering and Computer Science, The University of Michigan-Ann Arbor. He has 20 years of experience with wave propagation in random media, communication channel modeling, microwave sensors, and radar systems and is leading a large research group consisting of four research scientists, ten Ph.D. students, and two M.S. students. Over the past ten years he has graduated $20 \mathrm{Ph} . \mathrm{D}$. students. He has served as the Principal Investigator on many projects sponsored by NASA, JPL, ARO, ONR, ARL, NSF, DARPA and numerous industries. He has published many book chapters and more than 115 papers in refereed journals on electromagnetic scattering, random media modeling, wave propagation, antennas, microwave measurement techniques, radar calibration, inverse scattering problems, and microwave sensors. He has also had more than 230 papers and invited presentations in many national and international conferences and symposia on similar subjects. His research areas of interest include microwave and millimeter-wave radar remote sensing, electromagnetic wave propagation, and antenna miniaturization.
Dr. Sarabandi is a Member of the International Scientific Radio Union (URSI) Commission $\mathrm{F}$ and of The Electromagnetic Academy. He received the Henry Russel Award from the Regent of The University of Michigan-Ann Arbor (the highest honor the University of Michigan bestows on a faculty member at the assistant or associate level). He received a 1996 Teaching Excellence Award from the Department of Electrical Engineering and Computer Science and a 1999 GAAC Distinguished Lecturer Award from the German Federal Ministry for Education, Science, and Technology, given to about ten individuals worldwide in all areas of engineering, science, medicine, and law. He also received the 2003/2004 College of Engineering Research Excellence Award, The University of Michigan-Ann Arbor. In the past several years, joint papers presented by his students at a number of symposia (IEEE AP'95,'97,'00,'01,'03 IEEE IGARSS'99,'02, IEEE MTTS'01) have received student prize paper awards. He is a Vice President of the IEEE Geoscience and Remote Sensing Society (GRSS), a past Chairman of the Awards Committee of the IEEE GRSS from 1998 to 2002, and a Member of the IEEE Technical Activities Board Awards Committee from 2000 to 2002. He is an Associate Editor of the IEEE TRANSACTIONS ON ANTENNAS AND PROPAGATION and the IEEE SENSORS JOURNAL. He is listed in American Men \& Women of Science, Who's Who in America, and Who's Who in Electromagnetics.

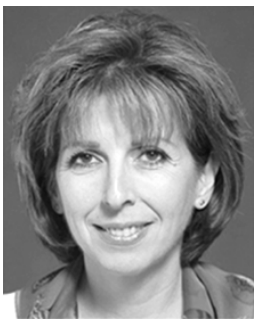

Linda P. B. Katehi (S'81-M'84-SM'89-F'95) received the B.S.E.E. degree from the National Technical University of Athens, Athens, Greece, in 1977 and the M.S.E.E. and Ph.D. degrees from the University of California, Los Angeles, in 1981 and 1984, respectively.

In September 1984, she joined the faculty of the Electrical Engineering and Computer Science Department of the University of Michigan, Ann Arbor, as an Assistant Professor and moved to the levels of Associate Professor in 1989 and Professor in 1994. She served in many administrative positions including: Director of Graduate programs in the College of Engineering from 1995 to 1996, Elected Member of the College Executive Committee from 1996 to 1998. Associate Dean for Graduate Education from 1998 to 1999 and Associate Dean for Academic Affairs from 1999 to 2001. In January 2002, she joined Purdue University as the John A. Edwardson Dean of Engineering and as Professor of Electrical and Computer Engineering. She has been the author or co-author of 450 papers published in referred journals and symposia proceedings and she holds five patents. She has graduated $25 \mathrm{Ph} . \mathrm{D}$. students.

Dr. Katehi is a Member of Sigma XI, Hybrid Microelectronics, the International Scientific Radio Union (URSI) Commission D, IEEE Antennas and Propagation Society (AP-S), and the Microwave Theory and Techniques Society (MTT-S). She has been awarded the IEEE AP-S W. P. King Best Paper Award for a Young Engineer in 1984, the IEEE AP-S S. A. Schelkunoff Award (Best Paper Award) in 1985, the NSF Presidential Young Investigator Award in 1987, the URSI Booker Award in 1987, the Humboldt Research Award in 1994, The University of Michigan Faculty Recognition Award in 1994, the IEEE MTT-S Microwave Prize in 1996, the International Microelectronics and Packaging Society (IMAPS ) Best Paper Award in 1997, the IEEE Third Millennium Medal in 2000, the IEE Marconi Prize in 2000, and the IEEE MTT-S Distinguished Educator Award in 2002. She was a member of AP-S ADCOM from 1992 to 1995 and she is serving currently on the IEEE MTT-S ADCOM. Also, she has been an Associate Editor for the IEEE TRANSACTIONS ON MICROWAVE THEORY AND TECHNIQUES and the IEEE TRANSACTIONS ON ANTENNAS AND PROPAGATION. 